\section{Ensayo sobre}

el uso de la encuesta:

hermenéutica $y$

reflexividad de la

\section{técnica investigativa}

\section{Francisco Ther Ríos*}

Claro que no se dirá, como querían los psicólogos, que lo objetivo ya es siempre subjetivo. Pero si se dirá que separado de lo subjetivo que lo porta y lo constituye 'intencionalmente', lo objetivo es abstracto, que oculta sus perspectivas, que es obra de un pensamiento ingenuo.

\section{Emmanuel} Levinas

Antropólogo, Maestro en Antropología Social. Docente e Investigador del Centro de Estudios del Desarrollo Local y Regional, Universidad de Los Lagos. Calle Lord Cochrane 1225, Osorno, Chile. E-mail: fther@ulagos.cl

\section{Abstract}

This essay focuses on the validity, prestige and excessive use of the survey, as a tool, to measure a social reality. Using the so called social research of second order, it is clear that this technique has limited social application. First, I proceed to characterize the survey and to correlate its essence with power and hidden domination, I debate the process of how the sample is extracted, how subjects are silent and missing. Then, I debate the likelihood of this approach and modeling of society. I concentrate on the rationalization, control, duality, occult, and silence and continuity of the social milieu.

\section{Resumen}

Este ensayo trata sobre el uso excesivo de la encuesta, su cotidianeidad, su prestigio en la sociedad, y su alcance como técnica diagnóstica de la realidad social. A través de la llamada investigación social de segundo orden se enuncia el poder invisible de esta técnica en lo social y se evidencia la restringida interpretación a que conduce su aplicación. Para tratar el tema, realizo una caracterización de la encuesta y relaciono su elaboración con el poder y la dominación soterrada; toco el proceso de extracción de la muestra, y el silencio y ausencia de los sujetos. En la segunda parte, hablo sobre la simulación de la sociedad a través de la modelación de la encuesta. Me remito entonces a la racionalización, control, dualidad, ocultamiento, silencio y continuidad de lo social. 


\section{Introducción: ¿cómo conocer?¹}

El interés del hombre por conocer y dominar su entorno se remonta al comienzo de la humanidad. Esta motivación esta dada por el deseo de controlar la manera en que se desenvolverán las actividades y acontecimientos de importancia como puede ser la organización social, la política, la economía y el mercado, los procesos eleccionarios, o las preferencias de los consumidores. En un comienzo el hombre se valía de fenómenos naturales para "leer" anticipadamente su futuro inmediato en las actividades de caza; las pinturas rupestres son prueba de esto. En las cuevas el hombre anticipaba la buena caza dibujando ceremonialmente en las paredes la muerte de la presa. Los oráculos en la antigüedad ejemplifican también el deseo por controlar lo desconocido. A través de ellos se deseaba conocer anticipadamente el futuro con el fin de corregirlo para el propio beneficio. Tan importante fue la figura de los oráculos que en ocasiones llegaron incluso a pronosticar la suerte de todo un reino. Lo común a estos y otros sistemas de creencias, es su deseo por anticipar el futuro a través de una visión o imagen. En la actualidad al hablar de pronósticos en seguida conjuramos imágenes de mujeres frente a bolas de cristal o métodos como los horóscopos en diarios. ¿Quiere decir esto que el hombre moderno no necesita ni busca ya controlar el futuro a través del presente? ¡No! La diferencia estriba en el tipo de oráculos que social y culturalmente se encuentran validados en el presente. De esta manera, las distintas investigaciones y formas de pensar sobre lo social siguen dando cuenta

Se sugiere revisar el Cuadro "Modelo Complejo para la Investigación Social", contenido en la "Introducción" del libro Niveles y Perspectivas de Investigación en la Ciencia. Diseños Investigativos. 2004. Francisco Ther Ríos (compilador). Diálogos Académicos del CEDER. Centro de Estudios del Desarrollo Local y Regional, Universidad de Los Lagos. Osorno, Chile. del mundo en que vivimos, al tiempo que nos acercan a distintas maneras de comprender aquello que denominamos como realidad para también mantener bajo control una imagen planificada del futuro. La intención parece seguir siendo la idea de anticiparnos a los eventos futuros en vez de padecerlos. Existiría así una similitud entre predicción y pronóstico. Ambas actividades son diferentes formas cognoscitivas para proyectar el futuro, resultando ser modelos de interpretación o normas de pensamiento de lo que está por-venir, pero al mismo tiempo guardan una importante diferencia. Mientras que la predicción nos remite a un único futuro, es decir, a cómo efectivamente se espera que acontezca el programa del futuro; el pronóstico, nos señala estratégicamente posibles escenarios para el futuro. El programa del futuro es racionalización de la sociedad por medio del modelo que se erige como omniabarcador. La racionalización equivale a la razón occidentalo-céntrica (MORIN, 1995: 104) cerrada, profundamente controladora y dominante. Por su parte, la orientación de posibles escenarios para el futuro emerge a través de la racionalidad abierta y reflexiva. La racionalidad "es el juego, el diálogo incesante, entre nuestro espíritu, que crea las estructuras lógicas, que las aplica al mundo, y que dialoga con ese mundo real" (MORIN, 1995: 102), de tal manera que la racionalidad es un "corrector del delirio lógico", del delirio de la coherencia absoluta impuesta por la modelación racionalizada y predictiva. Racionalización y racionalidad se encuentran y desencuentran; conviven, ocultándose una, apareciendo en cierto momento la otra. La interacción de ambas operatorias de la razón-del conocer- vistas como proceso que está siendo, constituye un desafío importante para las Ciencias Sociales y Humanas. Hacer el intento por entrar en esta atmósfera de climas contrapuestos y complementarios, de encuentros y desencuentros en lo cotidiano, 
equivale en el nivel metodológico a tener presente las potencialidades y limitaciones de las diversas técnicas que son correlato de la racionalización y la racionalidad. Predicción y pronóstico en tanto formas de ver y actuar sobre el mundo y el tiempo por-venir, pululan de distinta manera entre las cabezas, lo social y el control para oscilar eternamente en un banquete de ocultamientos, opacidad y develamientos. El desafío del cientista social es a instalarse en los procesos, y generar -activar- "algo". Ser conscientes de las potencialidades y límites de las técnicas de investigación permite entonces cierta conquista al otorgar un grado de libertad antes ignorado.

\section{Caracterización de la encuesta: interpretación y simulación de lo social}

Mientras que la hermenéutica nos permite interpretar a través de continuas comparaciones distintos enunciados interrelacionados por medio de sus significantes (formas) y significados (contenidos) -esto en un sentido gadameriano amplio como lo señala por ejemplo Ferraris (2000)-, la reflexividad -en el sentido que le otorga Ibáñez (1985, 1991) y Pask (1990), por ejemplo- nos remonta a un movimiento imaginativo volcado sobre el sujeto de la enunciación y el contenido del enunciado.

La hermenéutica, en tanto ejercicio, apunta hacia la búsqueda de significaciones y sentidos contextuales. Se trata de un cierto ejercicio de develamiento a partir de la comparación, nos pudiésemos preguntar entonces ¿qué sentido guarda el uso de la encuesta? Atrapados por el logos y el pensamiento, nos es imposible contar con una cosa distinta, incluso alternativa, a lo conocido y a la forma de conocer. Estamos atrapados en el lenguaje, el pensamiento es lenguaje y el logos se expresa en el lenguaje.
Sin embargo, esto no significa que todo redunde en lo mismo. Muy por el contrario, con el propio lenguaje podemos deconstruir-interpretar los núcleos operativos de la sociedad, regresando sobre ellos mismos para develar la gran expresión y valor agregado que contienen, y que no es posible de conocer de buenas a primeras por situar-se en un nivel algo más profundo de los modelos. Un ejercicio de interpretación y reflexividad permite entonces avanzar en el reconocimiento de las potencialidades y limitaciones de las acciones, develando lo que siempre ha estado ahí, medio escondido y a la vez muy presente. El sentido de la encuesta es de esta manera básicamente político. Como núcleo operativo de la sociedad impone el modelo que lo ha procurado en distinta intensidad, grado, forma y tiempo. Foucault (1996) remonta el origen de la encuesta al agrarismo feudal cuando el señor propietario feudal requería conocer el uso y destino de sus tierras; se clasificaban las tierras como aptas o no aptas para el cultivo, y dentro de las primeras se determinaba una subclasificación que venía a responder a los tipos particulares de cultivo. La tierra requería entonces de la fuerza de trabajo, la población se clasificaba -dividíaentre los campesinos capaces de cultivarla y aquellos que por edad y o enfermedad no podían hacerlo. Las tierras no aptas para el cultivo se destinaban para el ganado y vivienda. Estos principios de taxonomía agraria pasaron más tarde a los laboratorios, y desde allí a las Ciencias Sociales como técnica para ordenar a las poblaciones humanas (IBÁÑEZ 1991, 1998). Ordenar, clasificar, operaciones posibles de aplicar a lo anatómicamente similar, en lo social de nueva cuenta cierra lo diverso y anula lo extravagante para crear homogeneidad, creando una interpretación del "en sí, objetiva y objetivante. Con esta fuerza, la encuesta se transforma en núcleo operativo del modelo de 
sociedad, ella misma transforma a la sociedad. Su aplicabilidad en la sociedad capitalista y postindustrial es inmensa, basta ver su uso y sobreutilización a diario.

Desde el punto de vista de su estructura, la encuesta ${ }^{2}$ básicamente se construye a razón de preguntas abiertas y cerradas. Las últimas contienen categorías o alternativas de respuesta, pudiendo ser dicotómicas del tipo: SI - NO; o incluir varias alternativas: a, b, c, d, e. Aquí las categorías de respuestas son definidas por el investigador, quien responde elegirá por tanto entre las posibilidades dadas (ejemplo de esto son las escalas para medir actitudes). Por su parte, las preguntas abiertas no delimitan a priori las alternativas de respuesta, aunque si el ámbito de respuesta, no existiendo retroalimentación entre el encuestador y el encuestable. Teóricamente el número de respuestas en este tipo de encuestas es infinito, en la práctica no sucede esto pues existe una delimitación, un control no dicho por parte de quien hace y/o financia la encuesta. La selección de un tipo determinado de cuestionario exige también considerar algunos factores como son el grado de anticipación a las posibles respuestas, así como los tiempos para codificar las respuestas y la profundidad que se quiera conseguir en ella (HERNÁNDEZ, 1994: 289). De esta manera, las preguntas abiertas serán útiles, señala Roberto Hernández, cuando no se tenga información sobre las posibles respuestas o cuando aún teniendo información esta sea insuficiente; su desventaja es que "son difíciles de codificar, clasificar y preparar para su análisis". Las preguntas cerradas requieren por su parte de la anticipación a las posibles respuestas, siendo este tipo de cuestionario el más usado

Por mencionar algunos tipos de encuestas, existen las encuestas autoadministradas, por entrevista personal, por teléfono, autoadministradas y devueltas por correo, etc. por las ventajas que ofrece para quien investiga, ya que se estará en condiciones de disponer más fácilmente de los datos. De hecho es el investigador -que encuesta- quien lleva en su mente las respuestas de la encuesta, verdadera proyección de lo uno sobre lo múltiple, que aún muy informadamente (exhaustivo se dice) crea una atmósfera artificial de homogeneidad.

Una vez seleccionado el tipo de encuesta, la modelación de lo social requiere aplicar el instrumento a una parte del universo. El mapeo se realiza entonces sobre el doble operativo, simulando la presencia de algunos a los que artificiosamente se les permite la posibilidad de expresarse. La selección de la muestra implica operaciones casi implícitas: a saber, la extracción de la muestra teórica (o formamuestra) y la selección de la muestra empírica (o contenido-muestra). El muestreo se convierte entonces en un inconmovible supuesto lógico que se tiene como implícito en la elaboración de la encuesta y que, por tanto, no se cuestiona. Sin darnos cuenta de ello, la muestra exige la activación de "filtros" u operaciones que permitan trabajar con datos y construir en definitiva la modelación a partir de la determinación de la muestra. ${ }^{3}$ La primera de estas operaciones 0

\footnotetext{
Hay también las encuestas que trabajan con prácticamente toda la población en su conjunto (muestra = población), como los Censos. Estos básicamente siguen criterios demográficos orientados por necesidades de control político. Se necesita conoce para controlar. Control que se expresa en políticas públicas y sociales. Con este tipo de encuestas se simula una especie de mito fundador intrínseco que homogeniza la población, se informa sobre cifras, y se construye políticamente un individuo promedio. Si bien es cierto, existe información que los Censos recogen bien, como por ejemplo, información actualizada sobre cuántos somos, distribución por sexo, población económicamente activa, distribución etárea, niveles de desempleo, distribución y densidad poblacional, existe también una especificidad de sujetos de la cual los Censos no puede dar cuenta. Informa sólo una parte, un conjunto de dimensiones de la realidad, para luego trabajar con este fragmento como si fuese la realidad misma. Racionalización de las racionalidades y cierre de procesos.
} 
filtros se refiere a la forma-muestra, mientras que la segunda se orienta hacia el contenidomuestra. "La forma-muestra implica que un subconjunto de individuos (muestra) extraídos de su red de relaciones sociales representa al conjunto de los individuos (universo, población)" (IBÁÑEZ, 1998), existiendo aquí a lo menos dos algoritmias. En primer lugar, quien investiga traza fronteras artificiales para crear la muestra, convirtiendo en conjunto a una colección de individuos que se encuentran separados entre sí y, que no constituyen ningún "juntos" topológico o concreto, sólo son un conjunto abstracto. ${ }^{4}$ Desde este momento, el sistema manipulado por el investigador es presentado como un sistema organizacionalmente abierto, cuyos límites se encuentran dados artificialmente por la acción que significa el muestreo. En segundo término, la conformación de una muestra para la encuesta implica extraer-abstraer a los individuos que serán parte de la colección-muestra de sus respectivas redes de relaciones sociales, este cálculo ataña negativamente a lo que se pretende conocer y comprender, no lográndose vislumbrar las relaciones multidimensionales de tipo metafórico de las cuales el individuo es parte integrante a manera de un nudo que implica semejanza, pero no necesariamente contigüidad.

Una vez establecida la forma-muestra opera el segundo filtro sobre la población. Las llamadas "sustituciones" de los que teóricamente deben ser encuestados por los que pueden ser efectivamente entrevistados construyen una

\footnotetext{
Como también sucede con los individuos que ejercen su derecho al voto secreto en una elección política; aquí no interesa el sujeto votante con nombre y apellido. Interesa solo el individuo como elemento constituyente de la colección, es decir, interesa sumar 1 $+1+1+\ldots=$ Colección de Individuos = Colección de Votos.
}

gran distancia entre la muestra teórica y la empírica. Para que alguien sea ciertamente encuestado, la localización y la autorización del sujeto juegan un rol muy importante. Quienes presentan un movimiento browniano o irregular son más difíciles de encuestar, teniendo mayores probabilidades de ser entrevistables los individuos sedentes o que recorren una misma ruta cotidianamente. Quienes permiten mayoritariamente ser encuestados son los que están clasificados y dispuestos en posición de objetos y que pasan a constituir ciertamente las Ilamadas "mayorías silenciosas". Por el contrario quienes tienen derecho a la palabra, y los rebeldes que luchan por este derecho, dicen habitualmente "NO" a la posibilidad de responder a una encuesta. Todo esto revela "una sobrerrepresentación de los objetos (individuos) y una subrepresentación de los sujetos" silenciados. El contenido-muestra entonces definitivamente no logra llenar la forma-muestra (IBÁÑEZ, 1991: 140-148).

Los dos filtros que permiten y delimitan la extracción de la muestra hacen de la encuesta un dispositivo de control, relacionado con el silencio más que con el acto de hablar. El hablar posible en la encuesta es una simulación del acto de hablar verdadero. La encuesta desde sus orígenes ha cerrado la posibilidad de hablar; contrariamente a lo que se espera y piensa, este instrumento no deja hablar a los sujetos, sólo se habla a sí misma ante un espejo con forma de poder. Los juegos de lenguaje usados en la encuesta de pregunta / respuesta reproducen en este sentido las relaciones de poder. "Los que mandan pueden preguntar, los mandados deben responder" (IBÁÑEZ, 1991:12). El juego de control pregunta / respuesta (estímulo / respuesta) no logra hacer partícipe a quienes responden, las respuestas están contenidas ya en las preguntas. Se trata de 
una dualidad que no contiene redistribución ni del poder ni del deber; la encuesta, a pesar de hablar, no es una conversación. $Y$ no lo es justamente porque ella no es un intercambio de información entre unidades, entre personas, entre grupos, entre ideas o puntos de vista.

\section{Simulación y Modelación}

\section{II.1. Uso de la encuesta}

¿Quién no conoce una encuesta?. Probablemente, si no todos, la mayoría de nosotros nos hemos relacionado por una u otra razón con alguna encuesta. Ya sea participando en su elaboración rápida o sistematizada (y acorde con la metodología existente), ya sea por haber respondido una de ellas en algún momento, o por haberse negado a responderla, ya sea por la información que permite su análisis y que escuchamos y/o vemos en alguno de los medios de comunicación, o por cualquier otra razón, conocemos de la encuesta, y la validamos socialmente porque parece estar hablándonos de algo que alguien quiere conocer anticipadamente para controlar para el mejor beneficio propio. Pareciera que la información recogida por medio de una encuesta nos deja tranquilos, por ejemplo, "por lo bien que nos está yendo", o tan inquietos como para rápidamente tomar serias medidas y reorientar "nuestros planes":

Celebran el Día de Muertos 8 de cada 10 mexicanos: Considerada en el extranjero como una práctica 'valiente' de los mexicanos porque con ella retan a la 'temeraria y respetable' muerte, la conmemoración de 'Todos los Santos y los Fieles Difuntos' mantiene su vigencia, aún sobre 'la modernidad' y la tradición anglosajona del 'Halloween' (que se fortalece poco a poco en la capital del país). Los estratos sociales más bajos son los principales impulsores de esa creencia que raya entre lo religioso y lo laico, de acuerdo con una encuesta realizada por EL UNIVERSAL, en la que ocho de cada diez mexicanos aseguran cumplir con el rito de la ofrenda y con ella celebrar la 'llegada de sus muertos'.

México: Lee dos libros por año, el $56.2 \%$ de la población; $16.3 \%$, ninguno.

Casi la mitad de los habitantes del Distrito Federal (56.2\%) sólo lee uno o dos libros por año, mientras un $16.3 \%$ no lee ninguno; $23.5 \%$ lee entre tres y cinco; $8.2 \%$ lee entre seis y diez libros; $5.5 \%$ lee de 11 a 20 , y sólo un $1.5 \%$ lee más de veinte obras en ese mismo periodo, según los resultados de una encuesta levantada para EL UNIVERSAL sobre las preferencias culturales de las élites de la capital ... ${ }^{5}$

Discriminación en el trabajo (17.05.04).

Pregunta: ¿Crees que existe discriminación laboral en Chile?

Respuesta: SI = 3364 (94\%). NO = 174 (5\%). NO SÉ $=58(2 \%)$. Total $=3596$.

\section{Píldora del "Día Después" (13.04.04).}

Pregunta: ¿Estás de acuerdo con que se reparta la píldora del día después en los consultorios a mujeres que denuncien haber sido violadas?

Respuesta: $\mathrm{SI}=3751(84 \%)$. NO = $584(13 \%)$. NO SÉ $=112(3 \%)$. Total $=4447$.

Ley de Matrimonio Civil (12.03.04). ¿Estás de acuerdo con la aprobación de parte del Congreso de la Ley de Matrimonio Civil?

Respuesta: $\mathrm{SI}=2409$ (75\%). NO = $653(20 \%)$. NO SÉ $=167(5 \%)$. Total $=3229$.

Encuestas de la página de EL UNIVERSAL de México. Disponible en internet, noticias del 05 de marzo de 1999. http://serpiente. dgsc.unam.mx/universal/net1/encuestas/indice.html 
Encuesta Cerc sobre el progreso chileno (07.01.04). Un 55\% de los encuestados piensa que Chile está progresando y un $59 \%$ mira este año con esperanza.

Pregunta: ¿Estás de acuerdo con esta percepción?

Respuesta: $\mathrm{SI}=2135$ (69\%). NO = 794 (26\%). NO SÉ $=176(6 \%)$. Total $=3105$.

\section{Campaña contra el Sida (02.12.03).}

Pregunta: ¿Estás de acuerdo con la decisión de algunos canales de televisión de no difundir los spots del Sida?

Respuesta: $\mathrm{SI}=470$ (23\%). NO = $1545(75 \%)$. NO SÉ $=41(2 \%)$. Total $=2056$.

Discriminación en Chile (08.10.03). Estudio reveló que existen niveles preocupantes de intolerancia hacia etnias y pobres en Chile.

Pregunta: ¿Estás de acuerdo con la afirmación?

Respuesta: $\mathrm{SI}=1769$ (87\%). NO = $218(11 \%)$. NO SÉ $=51(3 \%)$. Total $=2038 .{ }^{6}$

"Un alto porcentaje de chilenos, el $37 \%$, señala haber vivido personalmente, o algún miembro de su familia, experiencias de discriminación, según una encuesta realizada por la Fundación Chile 21". ${ }^{7}$ En el estudio titulado Discriminación y desigualdades, los grupos más discriminados de la sociedad chilena son los indígenas y los pobres, seguidos a una cierta distancia por los viejos, los trabajadores y las mujeres. Ficha Técnica:

- 606 casos, encuesta telefónica.

- Aplicada en 10 ciudades: Santiago, Iquique,

De: http://encuestas.123.cl/anteriores.php?id=20

\footnotetext{
7 De: http://www.latercera.cl/articulo/0,0,3255_5666_77980424,00.
}

Antofagasta, La Serena, Coquimbo, Viña del Mar, Valparaíso, Concepción, Talcahuano y Temuco.

- Dirigida a hombres y mujeres mayores de 18 años

- Nivel de confianza: 95\%.

\section{II.2. Racionalización y efectos de la encuesta}

Las cifras que recogen las aplicaciones de encuestas simulan recoger información de la población general. Se simula el universo a través de muestras, devolviéndose a la población los resultados recogidos para darle forma a la sociedad. Se trata de la suplantación de lo real que racionaliza, reduce y simplifica por medio de algoritmos. Si el objetivo de la encuesta es conocer, y conocer justamente equivale en este contexto a clasificar y ordenar, tendremos que la encuesta es el instrumento de aplicación por excelencia en la investigación social para describir-prescribir.

Con las encuestas se clasifica y ordena. Las encuestas, entre otras cosas, informan acerca de cifras, de porcentajes a favor o en contra, de niveles sociales altos y bajos, de una separación por sexos, de preferencias políticas, o de opiniones. Pero también las encuestas crean opinión en la sociedad. Estas opiniones no son "meramente deducidas sino producidas. La encuesta ha dejado de ser un dispositivo de mera recolección de hechos a ser un dispositivo de producción de opiniones. Ya no se limita a extraer información (informarse de), sino que empieza a inyectar neguentropía (dar forma a)" (IBAÑEZ, 1991: 138). 
La encuesta entonces en sus múltiples variantes tanto informa como calla. Produce efectos, crea opiniones y permite informadamente que políticamente se decida. Sus resultados se devuelven a la población como características inmóviles propias. "El pueblo disfruta incluso día a día, como en un cine a domicilio, de las fluctuaciones de su propia opinión en la lectura cotidiana de los sondeos" (BAUDRILLARD, 1987: 143). ${ }^{8}$ Ir a la bases de la encuesta, es decir, a la posibilidad cierta o no de conocer que otorga la encuesta en tanto técnica de investigación, a sus potencialidades y limitaciones, significa así poner en practica cierto ejercicio de develamiento de las estrategias de poder en el saber.

La encuesta resulta ser un dispositivo de control. Con ella la sociedad de individuos es posible de ser sondeada, conocida, imaginada, simulada. Encuestas, test, sondeos, referéndum son dispositivos (del latín dispositus, dispuesto) de control, es decir, mecanismos que se disponen para obtener resultados automáticos. "Todos los sistemas actuales funcionan sobre esa entidad nebulosa, sobre esa sustancia flotante cuya existencia ya no es social, sino estadística, y cuyo único modo de aparición es el del sondeo. Simulación en el horizonte de lo social, o más bien en el horizonte donde lo social desapareció" (BAUDRILLARD, 1987: 127-128). Por definición, la encuesta es y será una técnica pragmáticamente rica, pero semánticamente pobre. "Aunque no se justifique teóricamente, se justifica prácticamente. Contribuye a

\footnotetext{
Los resultados de una encuesta a lo menos producirán un rechazo en el individuo que se informa (pero de ningún modo le serán del todo indiferente), y a lo más lo predispondrán a una actitud, generalmente positiva, hacia la información que se entrega con la encuesta. Generalmente, se espera que con los resultados de la encuesta el individuo receptor de la información tome partido y se sume irreflexivamente a la mayoría.
}

hacer que las cosas sean como se dice que son". (IBAÑEZ, 1991: 132-133). La encuesta contribuye a transformar la sociedad en un artificio, en una máquina mecánica. Con su uso excesivo ya ni siquiera se trata de imitar a la sociedad; los resultados que se consiguen no son ni la reiteración ni la parodia de la sociedad, sino una suplantación de lo real a través de signos de lo real; son, como dice Baudrillard, una operación de simulación de lo real a través del "doble operativo" (BAUDRILLARD, 1987: 11). Efectivamente la simulación, a diferencia de la disimulación, nos remite a la ausencia, a la reversión y eliminación de toda referencia. Simular es pretender ser "algo" que no se es, es tener "algo" que no se tiene. El ser y el tener viven aquí en la apariencia.

\section{II.3. Visualización y manejo de lo social}

La encuesta en tanto metodología estadística fue inventada para investigar un sistema que cumple más o menos con las condiciones de un "gas perfecto".

Un 'gas perfecto' es un buen modelo de gas: El gas es casi así, sus moléculas son casi libres y casi idénticas. El otro extremo es el cristal: En el gas dominan los elementos, en el cristal la estructura. La sociedad está más estructurada que un gas, pero menos que un cristal" (IBÁÑEZ, 1991: 132).

El simulacro convierte en piezas de museo, objetivables, a los individuos homogenizados. La sociedad se aparece como un conjunto de elementos idénticos e intercambiables. El museo en vez de circunscribirse a un reducto geográfico localizado, aparece por todos los lugares como una dimensión más de la vida. La generación de modelos de algo real se da en la 
extensión del museo en las demás esferas de la vida, como sucede con la encuesta que sobrepasa la cifra original simulando la existencia de sujetos que están ausentes. Importará pues más la encuesta con sus resultados que la sociedad encuestada, importará más el museo que los contextos culturales a los cuales remite. De esta manera, es innegable el fundamento ideológico y extremadamente parcelario, y no teórico, de la encuesta.

Una breve historia de Borges (1981: 143-144) manifiesta este deseo ideológico de modelar lo real:

En aquel Imperio, el Arte de la Cartografía logró tal Perfección que el mapa de una sola Provincia ocupaba toda una Ciudad, y el mapa del Imperio, toda una Provincia. Con el tiempo, esos mapas Desmesurados no satisficieron y los Colegios de Cartógrafos levantaron un Mapa del Imperio, que tenía el tamaño del Imperio y coincidía puntualmente con él. Menos Adictas al Estudio de la Cartografía, las Generaciones Siguientes entendieron que ese dilatado Mapa era inútil y no sin Impiedad lo entregaron a las Inclemencias del Sol y de los Inviernos. En los desiertos del Oeste perduran despedazadas Ruinas del Mapa, habitadas por Animales y por Mendigos; en todo el País no hay otra reliquia de las Disciplinas Geográficas. ${ }^{9}$

Suárez Miranda: Viajes de varones prudentes, libro cuarto, cap. XIV, Lérida, 1658.

Simulacro de la Ciencia por la técnica. La modelación o mapeo no tiene nada que ver con la pretendida realidad, esta es demasiada basta e incierta para ser miniaturizada. La modelación de la realidad es algo distinto a la misma

La modelación es racionalización de la sociedad, es un mapeo imposible de conseguir, pero que de igual manera se realiza, siempre con interés político. realidad. El deseo de aprehender la realidad no es más que deseo, es un simulacro, una especie de hiperreal o espacio sin atmósfera. Con la encuesta como medio para construir modelos sociales, el sujeto (individual, colectivo) deja de existir, el modelo liquida todos los referentes (BAUDRILLARD, 1987: 11). Nos informamos de una sociedad sin sujetos. Hablaremos así de una sociedad de individuos idénticos, perfectamente intercambiables entre sí y, por tanto, posibles de ser inventariados, clasificados, ordenados, atestiguados, constatados y conocidos. Ibáñez señala que la encuesta "es un procedimiento autoritario para producir la verdad. Se funda, según Foucault, en el derecho del poder soberano para establecer la verdad 'constatada' (constatación de algo no hablante, de un objeto) o 'atestiguada' (testimonio por algo hablante, meramente indicativo, de un hecho) 'mediante un cierto número de técnicas regladas"' (IBÁÑEZ, 1991: 135).

\section{Palabras finales: hacia el juego dialógico}

Concretemos algunos puntos:

Primero; la encuesta se instrumentaliza con el fin de "controlar" y hasta cierto punto predecir un futuro más o menos inmediato.

Segundo; la encuesta se articula sobre la base de una modelación de lo social, modelación efectuada a priori por quien investiga. Se extraen muestras que se devuelven como opinión de toda la sociedad. La encuesta por sobre todo ya no recoge opiniones, sino que es un instrumento que produce opiniones, dando con ello forma a lo social.

Tercero; en la extracción de la muestra, el contenido-muestra (muestra empírica) no logra llenar la forma-muestra (muestra teórica), 
cuestión que deja patente la reducción de lo social aún antes de aplicarse este dispositivo.

Cuarto; la sobreutilización de la encuesta en distintas esferas anula la pretendida recolección de datos, o de opiniones de sujetos, sólo refleja una acción violenta de exclusión de los sujetos.

En este sentido, el control que trata de imponerse por medio de la modelación produce opiniones, dando con ello forma a lo social y reduciéndolo a través de una acción violenta de exclusión de sujetos. La encuesta habla de sujetos sin historia, de individuos. Individuos que son perfectamente idénticos e intercambiables, "conocidos" a través de su falta de identidad. Se abre la posibilidad de conocer no lo que se pretende comprender, sino más bien validar un simulacro de la sociedad. El individuo cerrado sobre sí mismo, inventariado desde el control, no logra participar en el poder. El intercambio de poder y de deber no existe en la sociedad regulada por técnicas como la encuesta, sólo se dispone de una regulación y réplica tautológica del poder mediante el juego pregunta / respuesta.

El sujeto (individual y colectivo), en cambio se abre a la diversidad y exige en ella su reconocimiento. Percepciones, formas de vivir y habitar, anhelos y aspiraciones, experiencias, se erigen como formas recursivas de distribución del poder por medio de técnicas investigativas alejadas de la estructura oxidante de las encuestas y cuestionarios, deviniendo en una interlocución, no entre entidades, sino entre sujetos posibles de transformarse reflexivamente en actores. ${ }^{10}$

La sociedad de individuos resulta ser el contenedor de diversas prácticas y estrategias de poder, las cuales retroactúan sobre la primera para fortalecerla. O dicho de otra manera, la racionalización se vuelca en la cotidianeidad por medio de técnicas que nosotros mismos ayudamos tristemente a mantener.

10 En un nivel superior de la investigación social se encuentra el grupo de discusión, básicamente se trata de una conversación que representa a la sociedad de sujetos. También existe delimitación en cuanto a la población (o más bien muestra), pero aquí a diferencia de lo que sucede en la encuesta el dispositivo de control de ha distendido, el consultado es también consultante, existe una conversación. Es un proceso, en el cual sus miembros al conversar cambian, como cambia el sistema en que conversan. Para el grupo de discusión ver Jesús Ibáñez, Más allá de la sociología. El grupo de discusión: teoría y crítica, editorial Siglo XXI, Madrid, 1979. 


\section{Bibliografía}

Baudrillard, Jean (2000), Las Estrategias Fatales, Anagrama, Colección Argumentos, Barcelona.

Baudrillard, Jean (1987), Cultura y Simulacro, Editorial Kairós, Barcelona.

Borges (1981), "Del rigor en la Ciencia", en El Hacedor, Alianza Editorial, Madrid. Madrid.

Ferraris, Mauricio (2000), La Hermenéutica, Editorial Taurus,

Foucault, Michel (1999), Estrategias de poder, introducción, traducción y edición a cargo de Julia Varela y Fernando Álvarez, Edit. Paidós, España.

Foucault, Michel (1996), Vigilar y Castigar. Nacimiento de la prisión, Siglo XXI editores, España.

Foucault, Michel (1986), "Por qué hay que estudiar el poder: la cuestión del sujeto" en Materiales de Sociología Crítica, Wright Mills et al, Piqueta, Madrid.

Hernández, Roberto et al (1994), Metodología de la Investigación, Mc Graw-Hill, Colombia.

Ibáñez, Jesús (coord.) (1998), Nuevos avances en la investigación social II. Proyecto A ediciones, Barcelona.

Ibáñez, Jesús (1991), El regreso del sujeto. La investigación social de segundo orden. Edit. Amerinda, Chile.

Ibáñez, Jesús (1985), Del algoritmo al sujeto. Perspectivas de la investigación social, Siglo XXI, España.

Morin, Edgar (1996), Introducción al Pensamiento Complejo, Gedisa Editorial, Barcelona.
Morin, Edgar (1995), Sociología, Editorial Tecnos, Madrid.

Pask, Gordon (1990), "Relativismo", en Suplementos de la Revista Anthropos Nuevos avances en la investigación social. La investigación social de segundo orden, introducción y selección de textos de Jesús Ibáñez, Textos de la Historia Social de Pensamiento, $\mathrm{N}^{\circ} 22$, Barcelona.

Perec, Georges (1986), Pensar Clasificar, Editorial Gedisa, Barcelona.

Pérez-Taylor, Rafael et al (2000), Aprender-comprender la antropología, CECSA Editorial, México.

Ther, Francisco (2003), "Deconstruyendo al desarrollo. La Antropología del Límite: una propuesta de intervención teóricometodológica"; en Antropología y Estudios Regionales. De la aplicación a la acción, Colección LIDER, Centro de Estudios del Desarrollo Local y Regional / Universidad de Lagos, Chile.

Ther R., Francisco (1999), "Tipos de memoria social: niveles y perspectivas de análisis acerca del sujeto", ponencia presentada en el I Coloquio Nacional de Antropología y Psicología, INAH y Centro ELEIA de actividades psicológicas, Ciudad de México, Septiembre.

Ther R., Francisco (1999), "Sociedad sin sujetos, sociedad de individuos. El uso de la encuesta como dispositivo de control en la sociedad contemporánea", ponencia presentada en Segundo encuentro: La importancia de las ciencias sociales en la educación, sociedad y empresa, Instituto Politécnico Nacional, Ciudad de México, Mayo. 
$\underline{\underline{28}}$ 